\title{
Genome-wide identification of cold responsive transcription factors in Brassica napus L
}

\author{
Liping $\mathrm{Ke}^{1+}$, Weixia $\mathrm{Lei}^{2+}$, Weiguang Yang ${ }^{1}$, Jinyu Wang ${ }^{3}$, Janfang Gao ${ }^{1}$, Jinhua Cheng ${ }^{1}$, Yuqiang Sun',
} Zhixiong Fan ${ }^{2^{*}}$ and Dongliang $\mathrm{Yu}^{1^{*}}$

\begin{abstract}
Background: Cold stress is one of the primary environmental factors that affect plant growth and productivity, especially for crops like Brassica napus that live through cold seasons. Till recently, although a number of genes and pathways involved in B. napus cold response have been revealed by independent studies, a genome-wide identification of the key regulators and the regulatory networks is still lack. In this study, we investigated the transcriptomes of cold stressed semi-winter and winter type rapeseeds in short day condition, mainly with the purpose to systematically identify the functional conserved transcription factors (TFs) in cold response of B. napus.

Results: Global modulation of gene expression was observed in both the semi-winter type line (158A) and the winter type line $(\mathrm{SGDH} 284)$ rapeseeds, in response to a seven-day chilling stress in short-day condition. Function analysis of differentially expressed genes (DEGs) revealed enhanced stresses response mechanisms and inhibited photosynthesis in both lines, as well as a more extensive inhibition of some primary biological processes in the semi-winter type line. Over 400 TFs were differentially expressed in response to cold stress, including 56 of them showed high similarity to the known cold response TFs and were consistently regulated in 158A and SGDH284, as well as 25 TFs which targets were over-represented in the total DEGs. A further investigation based on their interactions indicated the critical roles of several TFs in cold response of B. napus.

Conclusion: In summary, our results revealed the alteration of gene expression in cold stressed semi-winter and winter ecotype B. napus lines and provided a valuable collection of candidate key regulators involved in B. napus response to cold stress, which could expand our understanding of plant stress response and benefit the future improvement of the breed of rapeseeds.
\end{abstract}

Keywords: Cold stress, Transcription factor, Brassica napus, Short day

\section{Background}

Rapeseed (Brassica napus L.) is one of the most important economic crops in the world, with three main ecotypes, i.e., spring-, winter- and semi-winter types, are formed in the scenario of evolution and long-term cultivation to adapt to the diverse climates. Yangtze River basin is the most important ecological region for rapeseed production

\footnotetext{
* Correspondence: zhixiongfan@126.com; yudl@zstu.edu.cn

${ }^{+}$Liping Ke and Weixia Lei contributed equally to this work.

${ }^{2}$ Crop Institute, Anhui Academy of Agricultural Sciences, Hefei 230031, China

'Plant Genomics \& Molecular Improvement of Colored Fiber Lab, College of Life Sciences and Medicine, Zhejiang Sci-Tech University, Hangzhou 310018, China

Full list of author information is available at the end of the article
}

in China. Winter- and semi-winter rapeseeds are cultivated in Yangtze River basin with the seeds sowed from the end of September to mid-October, which means the growth and production of rapeseed are always threatened by the cold temperature. Therefore, determining the molecular mechanisms that rapeseeds have evolved to winter survival would not only make us understand more about the plant environmental adaptation but is also of great agricultural importance for China and other countries.

Cold acclimation, i.e., a period of exposure to low but non-freezing temperature, would increase the frost tolerance in a wide range of plants [1]. In this process, a series of plant biochemical and physiological features are adjusted

(c) The Author(s). 2020 Open Access This article is distributed under the terms of the Creative Commons Attribution 4.0 International License (http://creativecommons.org/licenses/by/4.0/), which permits unrestricted use, distribution, and reproduction in any medium, provided you give appropriate credit to the original author(s) and the source, provide a link to the Creative Commons license, and indicate if changes were made. The Creative Commons Public Domain Dedication waiver (http://creativecommons.org/publicdomain/zero/1.0/) applies to the data made available in this article, unless otherwise stated. 
and large-scaled alteration of gene expression are induced. Over the few past decades, great efforts have been made to unravel the molecular mechanisms involved in plant cold acclimation. CBFs/DREBs (cold-binding transcription factors/dehydration responsive element binding factors) -dependent signaling, the key and conserved regulatory mechanism of cold stress response, was characterized in many plants, with the components containing the CBFs and their activators (e.g., ICE1, CAMTA3 and BZR1/BES1) and repressors (e.g., MYB15, PIFs and EIN3) [2, 3]. Meanwhile, involvement of some CBF-independent regulatory pathways in cold response was also characterized in many plants, such as the plant hormones of auxin, abscisic acid, ethylene, gibberellins and jasmonic acid [4].

To date, many studies have investigated the physiobiochemical and molecular changes of cold stressed rapeseeds. In cell ultrastructure, pronounced modifications were observed in cold acclimation of B. napus, such as increased thickness of cell walls and invagination of plasma membranes [5]. Besides, extensive modulation in gene expression was also detected, including transcript accumulation for an arrange of genes, e.g., the cold-responsive (COR) genes BN115 (COR15) and BNCOR25 [6, 7], BN59 ( $\mathrm{H}^{+}$-ATPase subunit) [8], hsp 90 [9], multiple $C B F \mathrm{~s}[10,11]$ and a series of transcription factors (TFs) like APETALA2/ethylene response factors and NACs [12, 13]. More recent studies also indicated the potential roles of microRNAs and lncRNAs in rapeseed response to cold stress $[14,15]$. However, there are still quite many remaining entangled, e.g., most of the previous researches were implemented in long-day photoperiod conditions, which were divorced from the short day condition in actual field production. Moreover, it remains unclear if the known mechanisms are conserved across accessions and in which manner those COR genes are regulated.

In the present work, we comparatively analyzed the transcriptomes of cold stressed semi-winter (158A) and winter type (SGDH284) rapeseeds after a seven-day cold treatment, with the purpose to reveal the conserved and key regulators in the cold response of rapeseed under short-day photoperiod.

\section{Results}

\section{Identification and general features of differentially expressed genes (DEGs)}

High-throughput sequencing generated an average of about 6.5 million qualified short reads from each samples of the semi-winter type (158A) and the winter type (SGDH284) $B$. napus lines, with around $70 \%$ of them were mapped to the reference genome (Additional file 1: Table S1). With the cutoff of FPKM $\geq 1$ in at least one samples, expression of about 40,000 genes ( $25 \%$ of the whole set) were detected in 158A $(40,277)$ and SGDH284 $(39,200)$. Notably, a greater number of expressed genes were detected in cold stressed $158 \mathrm{~A}$ and SGDH284 than the controls, with the ratios of about 8.6 and $11.6 \%$, respectively.

After exposure to low temperature, about $10 \%$ of the whole set of genes $(\sim 10,000$ genes) were differentially expressed in both 158A and SGDH284, including 2436 and 3306 genes that were consistently up- and downregulated in both lines (Fig. 1a). Function enrichment

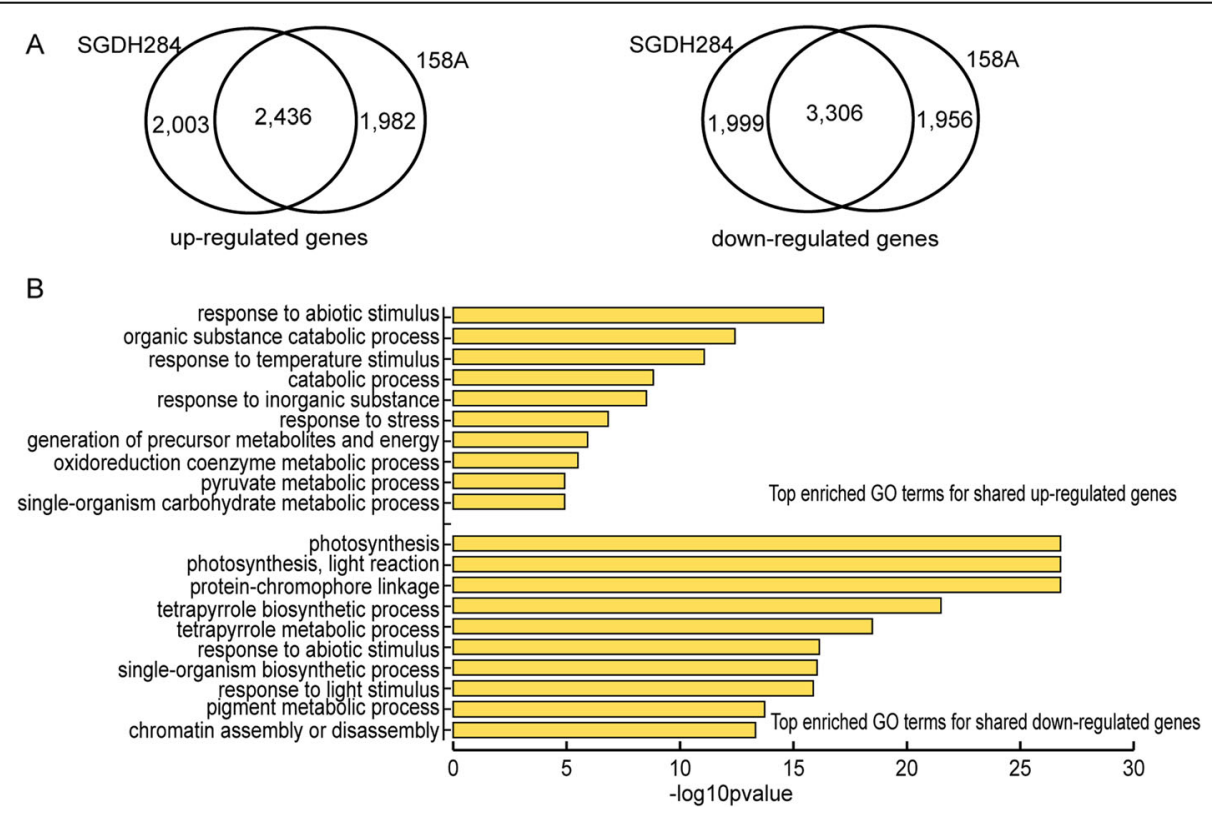

Fig. 1 Differentially expressed genes in cold stressed Brassica napus. a Venn diagrams indicated the number of differentially expressed genes in 158A and/or SGDH284. b Function analysis of shared up- and down-regulated genes. Top 10 enriched GO terms were showed 
analysis revealed that, the shared up-regulated genes were significantly enriched in mechanisms responsible for a wide range of environmental stresses and the catabolic processes related to energy production, while the shared down-regulated genes were enriched in biological processes associated with photosynthesis, stress response and chromatin organization (Fig. 1b). Functions of the line-specific DEGs were also analyzed, which revealed the remarkable differences in gene expression between cold stressed 158A and SGDH284. In the semi-winter line 158A, the down-regulated genes were significantly associated with the processes such as organonitrogen compound biosynthesis, microtubule-based movement, translation and DNA replication, whilst the up-regulated genes were mainly related to the stresses response and transporters. In contrast, down-regulated genes in the winter type line SGDH284 were mainly involved in stresses response and amino acid/sugar/sulfur compound metabolic processes, and the SGDH284 specific upregulated genes were enriched in the processes related to the biosynthesis of constitute macromolecules and histone ubiquitination (Table 1). Further investigation revealed that significantly more genes involved in translation, DNA replication and regulation of cell cycle were identified in 158A specific down-regulated genes, as well as more genes associated with the ribosome biogenesis were found in the SGDH284 specific up-regulated gene set (Additional file 1: Table S2), suggesting the basic biological processes that maintained the cellular activities were more significantly inhibited in the semi-winter line 158A.

Some previously characterized COR genes in B. napus were found in the collected DEGs in this work. BN28 (BnaAnng37980D, Kin1), BN115 (BnaA03g56750D, COR15) and BN59 (BnaA07g34490D, VHA-A) were the first characterized COR genes in $B$. napus, with their transcripts accumulated in cold stressed plants $[6,8,16]$. In this work, up-regulation of these genes were observed in both 158A and SGDH284, with the log2foldchanges ranged from 1.1 to 5.6. Earlier studies also revealed transcripts accumulation of four CBF-like genes in $B$. napus response to low temperature, e.g., $C B F 5$, CBF7, CBF16 and CBF17 [10, 11]. CBF5 (BnaC03g71900D), CBF7 (BnaAnng34260D) and CBF16 (BnaC07g39680D) were also up-regulated during low-temperature exposure of 158A and SGDH284 (log2foldchange ranged from 2.4-4.4). However, the exact locus for $C B F 17$ was not determined in the selected genome version (PRJEB5043_v1), which might be ascribed to the difference in gene model construction during genome annotation. The most similar gene to CBF17 was BnaA08g30910D (80\% identity), but it was not varied in expression in any cold stressed lines in this work.

In contrast, transcripts accumulation for some other known COR genes might not be essential for cold acclimation of 158A or SGDH284 in short-day condition.
For example, previous reports revealed that $B n C O R 25$ and $h s p 90$ were induced in B. napus by cold stress $[7,9]$. However, transcriptomics analysis in this work found that the expression of $B n C O R 25$ or $h s p 90$ was not significantly altered in either 158A or SGDH284 after low temperature exposure.

\section{Expression of TFs in cold stressed $B$. napus}

TFs play crucial roles in plant response to various environmental stresses, e.g., in the well characterized model organism A. thaliana, more than a thousand stress responsive TFs were identified based on the integrated curation and genomic data mining approach [17]. In $B$. napus, a total of 5985 TFs were annotated, with many of them involved in stresses response, such as the CBFs and a series of NACs $[10,18]$. However, a systematic identification of cold stress responsive TFs is still lack.

In this work, derived from the estimated expression profiles of two $B$. napus lines, we found that expression of about $40 \%$ of the TFs were detectable (FPKM $\geq 1$ ) in at least one of the investigated samples. 692 and 708 TFs were differentially expressed during low-temperature exposure of 158A and SGDH284, respectively, including 402 TFs shared by two lines. All the differentially expressed TFs belonged to 54 families (Additional file 1: Table S3 and Fig. 2). Of them, bHLH was the largest family that about 70 differentially expressed bHLH type TFs were identified in each line. However, it was COlike family that had the highest ratio (about 50\%) of varied expressed members. Interestingly, in the collected differentially expressed TFs, members of some families were mostly down-regulated (e.g., bHLH, GATA and ZF-HD) or up-regulated (e.g., WRKY, C3H and DBB) in both lines. Specifically, all of the differentially expressed WRKY (35 genes) were up-regulated in 158A, in contrast to that both up-regulated (14 genes) and down-regulated (8 genes) WRKY genes were found in SGDH284. In addition, in both 158A and SGDH284, almost all the differentially expressed GRFs (growth-regulation factors) were down-regulated.

\section{Identification of key regulators involved in cold response}

Two strategies were used to identify the key regulators involved in B. napus response to cold stress. First, homology search identified 128 TFs that showed high sequence similarity ( $>50 \%$ identity in amino acid) to the cold-responsive TFs in A. thaliana and were differentially expressed in 158A or/and SGDH284 after plants exposure to low temperature. 19 and 37 of them were down- (e.g., multiple ethylene-responsive transcription factors) and up-regulated (e.g., homologs of CBF1/2 and ZAT6/10/12) in both 158A and SGDH284, respectively (Table 2). Derived from the description in STIFDB (Stress Responsive Transcription Factor Database) [17], 
Table 1 Function enrichment analysis of line-specific differentially expressed genes in cold stressed semi-winter (158A) and winter type (SGDH284) B. napus plants

\begin{tabular}{|c|c|c|c|}
\hline Class & Term_ID & Description & $\log 10(p$-value $)$ \\
\hline \multicolumn{4}{|c|}{ top10 enriched GO terms for line-specific down-regulated genes } \\
\hline \multirow[t]{10}{*}{$158 \mathrm{~A}$} & GO:1901566 & organonitrogen compound biosynthetic process & -12.42 \\
\hline & GO:1901564 & organonitrogen compound metabolic process & -11.23 \\
\hline & GO:0007018 & microtubule-based movement & -10.96 \\
\hline & GO:0006928 & movement of cell or subcellular component & -10.82 \\
\hline & GO:0007017 & microtubule-based process & -10.15 \\
\hline & GO:0006412 & translation & -8.25 \\
\hline & GO:0043603 & cellular amide metabolic process & -7.92 \\
\hline & GO:0044249 & cellular biosynthetic process & -7.08 \\
\hline & GO:1901576 & organic substance biosynthetic process & -6.82 \\
\hline & GO:0006270 & DNA replication initiation & -6.40 \\
\hline \multirow[t]{10}{*}{ SGDH284 } & GO:0051707 & response to other organism & -7.89 \\
\hline & GO:1901605 & alpha-amino acid metabolic process & -7.77 \\
\hline & GO:0044283 & small molecule biosynthetic process & -7.66 \\
\hline & GO:0046394 & carboxylic acid biosynthetic process & -7.52 \\
\hline & GO:0009605 & response to external stimulus & -7.43 \\
\hline & GO:0009607 & response to biotic stimulus & -6.55 \\
\hline & GO:0016998 & cell wall macromolecule catabolic process & -6.31 \\
\hline & GO:0046348 & amino sugar catabolic process & -5.92 \\
\hline & GO:0044710 & single-organism metabolic process & -5.62 \\
\hline & GO:0006790 & sulfur compound metabolic process & -5.42 \\
\hline \multicolumn{4}{|c|}{ top10 enriched GO terms for line-specific up-regulated genes } \\
\hline \multirow[t]{10}{*}{$158 \mathrm{~A}$} & GO:0051707 & response to other organism & -9.28 \\
\hline & GO:0051704 & multi-organism process & -8.19 \\
\hline & GO:0009605 & response to external stimulus & -8.18 \\
\hline & GO:0009607 & response to biotic stimulus & -7.38 \\
\hline & GO:1901565 & organonitrogen compound catabolic process & -6.68 \\
\hline & GO:1902578 & single-organism localization & -5.62 \\
\hline & GO:0006855 & drug transmembrane transport & -5.51 \\
\hline & GO:0042493 & response to drug & -5.42 \\
\hline & GO:0055085 & transmembrane transport & -5.07 \\
\hline & GO:0044765 & single-organism transport & -4.74 \\
\hline \multirow[t]{10}{*}{$\mathrm{SGDH} 284$} & GO:0042254 & ribosome biogenesis & -9.72 \\
\hline & GO:0006396 & RNA processing & -7.68 \\
\hline & GO:0034470 & ncRNA processing & -6.64 \\
\hline & GO:0051503 & adenine nucleotide transport & -4.82 \\
\hline & GO:0030163 & protein catabolic process & -4.59 \\
\hline & GO:0044085 & cellular component biogenesis & -4.54 \\
\hline & GO:0007275 & multicellular organism development & -4.29 \\
\hline & GO:0009793 & embryo development ending in seed dormancy & -4.27 \\
\hline & GO:0033523 & histone $\mathrm{H} 2 \mathrm{~B}$ ubiquitination & -4.09 \\
\hline & GO:0016574 & histone ubiquitination & -4.09 \\
\hline
\end{tabular}




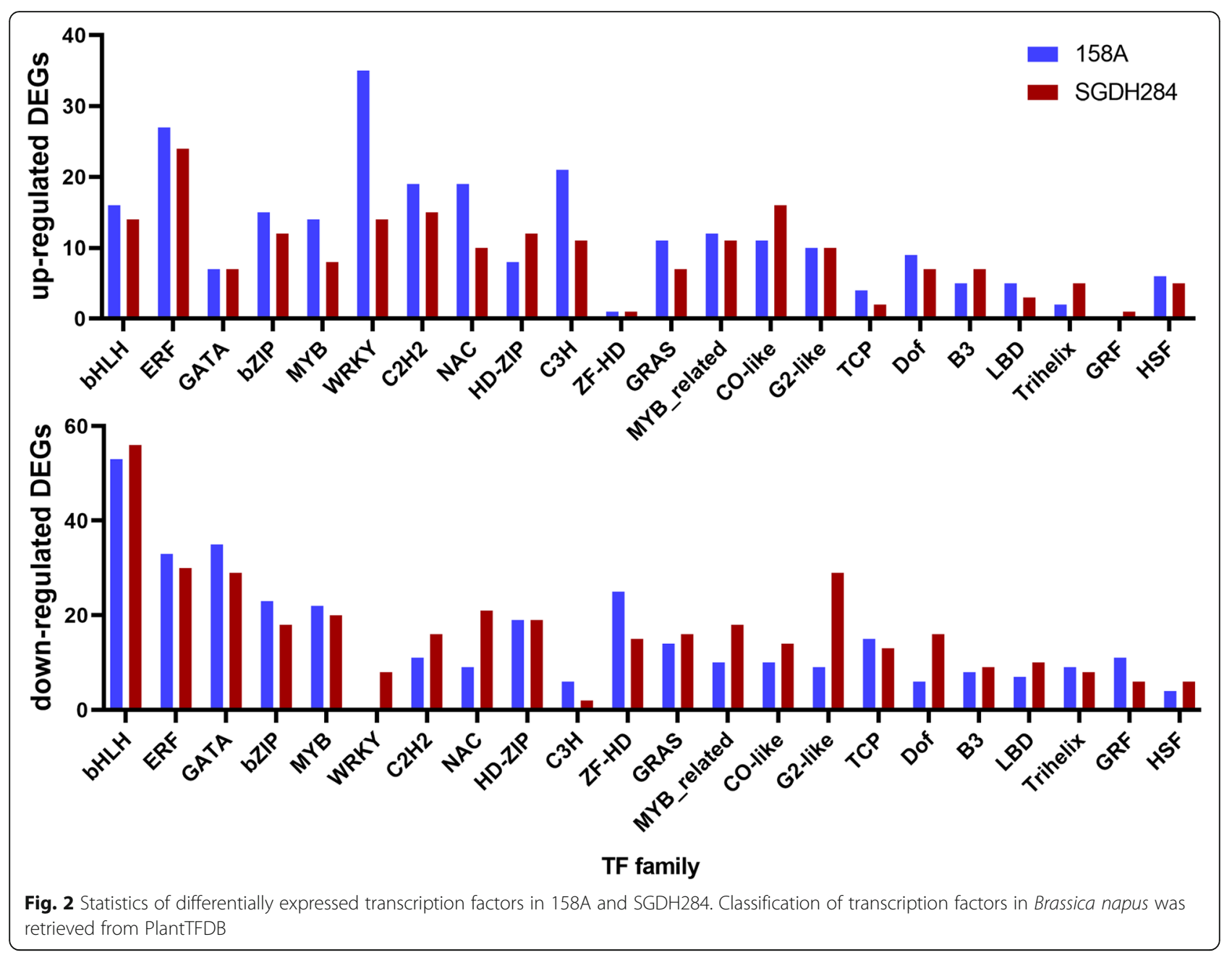

most of these 56 TFs were also involved in response to some other stresses, such as drought, light and salt.

In addition, enrichment analysis was carried out to find the TFs with their targets over-represented in the total DEGs, resulting in 51 and 55 enriched TFs in line 158A and SGDH284, respectively (Additional file 1: Table S4). Twenty-five of these TFs were shared by two lines (Table 3), including several genes homologous to COR genes in other plants, such as CBF7, CAMTA1 and PIF4/7 that were involved in the CBF pathway, RVE7 and TCP21 in the regulation of the circadian clock by interacting with the CBF pathway components LHY and CCA1 [19, 20], BZIP44 and CDF5 in seed germination and flowering $[21,22]$, as well as some other genes associated with multiple stresses response. Notably, 1495 and 1520 potential targets of these 25 TFs were differentially expressed in 158A and SGDH284, accounting for about $15 \%$ of the total DEGs, respectively. Function analysis revealed that 16 out of these 25 TFs were enriched in biological processes like photosynthesis, plant development (e.g., seed germination, root development and circadian rhythm), amino acid catabolism and stimulus response (Table 3 ).

Summarily, homology search and TF enrichment based strategies identified 56 and 25 TFs, respectively, which were up-/down-regulated in both 158A and SGDH284. Ten of them were selected and their expression variation under cold stress condition was validated by RT-qPCR (Fig. 3 and Additional file 1: Table S5).

Moreover, inferred from the curated protein-protein actions in $A$. thaliana and the predicted regulation in B. napus, a regulatory network of the identified cold-responsive TFs was built (Fig. 4). According to the constructed networks, some TFs worked at the initial steps of regulation, such as BnaAnng34260D (CBF7) and BnaC07g07840D (BHLH77), while some TFs regulated the expression of multiple other TFs, e.g., BnaA06g26010D (TCP7) and BnaC09g47560D (TCP21) and BnaA03g40080D (PIF7).

\section{Discussion}

In an attempt to further understanding the molecular mechanisms of $B$. napus response to cold stress, herein 
Table 2 Homology based identification of cold responsive transcription factors in Brassica napus

\begin{tabular}{|c|c|c|c|c|c|c|c|}
\hline \multirow[t]{2}{*}{ Gene in B. napus } & \multirow{2}{*}{$\begin{array}{l}\text { Homolog in } \\
\text { A. thaliana }\end{array}$} & \multirow[t]{2}{*}{ Symbol } & \multirow[t]{2}{*}{ Identity\% } & \multicolumn{2}{|c|}{ log2foldchange } & \multirow[t]{2}{*}{ Description } & \multirow[t]{2}{*}{ Stress elements } \\
\hline & & & & $158 \mathrm{~A}$ & $\mathrm{SGDH} 284$ & & \\
\hline BnaCnng41320D & AT3G19290 & ABF4 & 66.8 & 1.7 & 1.6 & $\begin{array}{l}\text { Abscisic acid responsive elements- } \\
\text { binding factor } 4\end{array}$ & drought/NaCl/ABA/cold \\
\hline BnaA05g11360D & AT2G31380 & $\mathrm{BB} \times 25$ & 88.7 & 2.7 & 1.6 & B-box zinc finger protein 25 & cold/light \\
\hline BnaCnng49280D & AT4G25490 & CBF1 & 65.6 & 5.2 & 7.2 & $\begin{array}{l}\text { Dehydration-responsive element- } \\
\text { binding protein 1B }\end{array}$ & cold/drought/salt \\
\hline $\begin{array}{l}\text { BnaAnng34260D } \\
\text { BnaC07g39680D } \\
\text { BnaC03g71900D } \\
\text { BnaA03g13620D }\end{array}$ & AT4G25470 & CBF2 & $\begin{array}{l}78.2 \\
74.2 \\
76.5 \\
77.0\end{array}$ & $\begin{array}{l}2.4 \\
3.2 \\
4.3 \\
2.4\end{array}$ & $\begin{array}{l}2.7 \\
3.4 \\
4.4 \\
6.9\end{array}$ & $\begin{array}{l}\text { Dehydration-responsive element- } \\
\text { binding protein } 1 \mathrm{C}\end{array}$ & cold/drought/salt \\
\hline BnaC04g31720D & AT5G39660 & CDF2 & 74.6 & 2.6 & 1.1 & Cyclic dof factor 2 & cold/drought/UV-B \\
\hline $\begin{array}{l}\text { BnaA02g02840D } \\
\text { BnaC09g41980D } \\
\text { BnaA10g18420D }\end{array}$ & AT5G15850 & COL1 & $\begin{array}{l}63.1 \\
66.6 \\
66.4\end{array}$ & $\begin{array}{l}2.2 \\
2.7 \\
2.2\end{array}$ & $\begin{array}{l}1.3 \\
1.5 \\
1.7\end{array}$ & Zinc finger protein CONSTANS-LIKE 1 & cold/ABA/drought/light/NaCl \\
\hline BnaC03g35440D & \multirow[t]{2}{*}{ AT3G07650 } & \multirow[t]{2}{*}{ COL9 } & \multirow{2}{*}{$\begin{array}{l}80.1 \\
85.9\end{array}$} & \multirow{2}{*}{$\begin{array}{l}4.2 \\
1.6\end{array}$} & \multirow{2}{*}{$\begin{array}{l}3.1 \\
4.5\end{array}$} & \multirow[t]{2}{*}{ Zinc finger protein CONSTANS-LIKE 9} & \multirow[t]{2}{*}{ cold $/ \mathrm{NaCl}$} \\
\hline BnaA05g29980D & & & & & & & \\
\hline BnaC03g22460D & AT2G40140 & CZF1 & 75.6 & 5.0 & 2.2 & $\begin{array}{l}\text { Zinc finger } \mathrm{CCCH} \text { domain - } \\
\text { containing protein }\end{array}$ & cold \\
\hline BnaC09g49920D & \multirow[t]{2}{*}{ AT5G05410 } & \multirow[t]{2}{*}{ DREB2A } & 64.7 & 1.8 & 2.9 & \multirow{2}{*}{$\begin{array}{l}\text { Dehydration-responsive element- } \\
\text { binding protein } 2 \mathrm{~A}\end{array}$} & \multirow[t]{2}{*}{ cold/drought/salt } \\
\hline BnaA10g25000D & & & 62.2 & 2.8 & 3.8 & & \\
\hline BnaA05g05080D & AT3G61150 & HDG1 & 65.0 & 1.1 & 1.2 & $\begin{array}{l}\text { Homeobox-leucine zipper } \\
\text { protein HDG1 }\end{array}$ & cold \\
\hline BnaCnng44540D & AT3G24310 & MYB305 & 81.8 & 7.1 & 5.2 & $\begin{array}{l}\text { MYB-like DNA-binding domain } \\
\text { protein }\end{array}$ & cold $/ \mathrm{NaCl}$ \\
\hline BnaA03g53110D & AT4G34990 & MYB32 & 75.4 & 1.4 & 2.3 & Transcription factor MYB32 & cold/NaCl \\
\hline BnaA01g16400D & AT4G27410 & NAC072 & 87.0 & 2.3 & 1.4 & NAC domain-containing protein 72 & cold/drought/NaCl/ABA/light \\
\hline BnaC08g04820D & AT1G46768 & RAP2-1 & 78.1 & 3.4 & 2.6 & $\begin{array}{l}\text { Ethylene-responsive transcription } \\
\text { factor RAP2-1 }\end{array}$ & cold \\
\hline BnaA05g25200D & \multirow[t]{3}{*}{ AT3G14230 } & \multirow[t]{3}{*}{ RAP2-2 } & 73.6 & 1.8 & 1.3 & \multirow{3}{*}{$\begin{array}{l}\text { Ethylene-responsive transcription } \\
\text { factor RAP2-2 }\end{array}$} & \multirow[t]{3}{*}{ cold } \\
\hline BnaC05g39400D & & & 74.1 & 1.8 & 1.6 & & \\
\hline BnaA03g33290D & & & 69.5 & 1.0 & 1.9 & & \\
\hline BnaC03g08310D & AT5G17300 & RVE1 & 78.0 & 2.7 & 1.9 & Protein REVEILLE 1 & cold/drought/salt \\
\hline BnaA01g19870D & \multirow[t]{2}{*}{ AT3G46600 } & \multirow[t]{2}{*}{$\mathrm{SCL} 30$} & 80.4 & 1.6 & 1.5 & \multirow[t]{2}{*}{ Scarecrow-like protein 30} & \multirow[t]{2}{*}{ cold/drought/UV-B/iron } \\
\hline BnaC01g24660D & & & 70.6 & 1.3 & 1.8 & & \\
\hline BnaA05g11640D & AT2G31070 & TCP10 & 76.8 & 1.9 & 2.0 & Transcription factor TCP10 & cold \\
\hline BnaC07g43320D & AT4G31550 & WRKY11 & 72.0 & 1.5 & 1.1 & Probable WRKY transcription factor 11 & ABA/cold/light \\
\hline BnaA04g22040D & AT2G38470 & WRKY33 & 75.9 & 4.0 & 1.8 & Probable WRKY transcription factor 33 & cold/drought \\
\hline BnaC03g58080D & \multirow[t]{2}{*}{ AT1G27730 } & ZAT10 & 87.6 & 1.7 & 2.2 & Zinc finger protein ZAT10 & cold/drought/ABA/NaCl \\
\hline BnaC07g16940D & & & 61.8 & 7.1 & 3.6 & & \\
\hline BnaA03g09250D & AT5G59820 & ZAT12 & 85.2 & 1.4 & 1.1 & Zinc finger protein ZAT12 & cold/drought \\
\hline BnaC09g35160D & & & 84.7 & 2.6 & 2.4 & & \\
\hline BnaA10g12780D & & & 86.4 & 3.4 & 3.7 & & \\
\hline BnaA10g25850D & AT5G04340 & ZAT6 & 76.0 & 2.3 & 3.7 & Zinc finger protein ZAT6 & cold \\
\hline BnaCnng20570D & & & 72.0 & 4.0 & 7.7 & & \\
\hline BnaA03g54460D & AT4G39780 & ERF060 & 76.2 & -2.7 & -3.4 & Ethylene-responsive transcription & cold \\
\hline BnaC07g46940D & & & 76.4 & -2.3 & -2.7 & & \\
\hline BnaA09g55520D & AT3G61890 & ATHB-12 & 78.9 & -2.1 & -2.0 & $\begin{array}{l}\text { Homeobox-leucine zipper } \\
\text { protein ATHB-12 }\end{array}$ & cold/drought/NaCl \\
\hline
\end{tabular}


Table 2 Homology based identification of cold responsive transcription factors in Brassica napus (Continued)

\begin{tabular}{|c|c|c|c|c|c|c|c|}
\hline \multirow[t]{2}{*}{ Gene in B. napus } & \multirow{2}{*}{$\begin{array}{l}\text { Homolog in } \\
\text { A. thaliana* }\end{array}$} & \multirow[t]{2}{*}{ Symbol } & \multirow[t]{2}{*}{ Identity\% } & \multicolumn{2}{|c|}{$\log 2$ foldchange ${ }^{\#}$} & \multirow[t]{2}{*}{ Description } & \multirow[t]{2}{*}{ Stress elements $^{* *}$} \\
\hline & & & & $158 \mathrm{~A}$ & $\mathrm{SGDH} 284$ & & \\
\hline BnaA05g24390D & \multirow[t]{4}{*}{ AT3G15210 } & \multirow[t]{4}{*}{ ERF4 } & 72.7 & -1.2 & -2.2 & \multirow{4}{*}{$\begin{array}{l}\text { Ethylene-responsive transcription } \\
\text { factor } 4\end{array}$} & \multirow[t]{4}{*}{ cold/drought } \\
\hline BnaA06g01090D & & & 64.7 & -2.5 & -2.0 & & \\
\hline BnaC03g39000D & & & 77.3 & -1.4 & -1.9 & & \\
\hline BnaA03g33790D & & & 76.4 & -1.2 & -1.8 & & \\
\hline BnaC05g37390D & \multirow[t]{4}{*}{ AT4G14540 } & \multirow[t]{4}{*}{ HAP3C } & 85.1 & -2.0 & -2.6 & \multirow[t]{4}{*}{ Transcriptional activator HAP3C } & \multirow[t]{4}{*}{ cold } \\
\hline BnaC03g39380D & & & 85.3 & -2.3 & -2.3 & & \\
\hline BnaA03g33970D & & & 78.3 & -1.9 & -2.1 & & \\
\hline BnaA01g28350D & & & 85.5 & -1.2 & -1.6 & & \\
\hline BnaC02g25060D & AT1G78600 & LZF1 & 83.7 & -2.2 & -2.1 & B-box zinc finger protein 22 & cold \\
\hline BnaC08g48630D & AT3G50060 & MYB77 & 72.1 & -1.4 & -1.0 & Transcription factor MYB77 & cold \\
\hline BnaA07g13990D & \multirow[t]{2}{*}{ AT2G28550 } & \multirow[t]{2}{*}{ RAP2-7 } & 80.9 & -1.7 & -1.9 & \multirow{2}{*}{$\begin{array}{l}\text { Ethylene-responsive transcription } \\
\text { factor RAP2-7 }\end{array}$} & \multirow[t]{2}{*}{ cold/ABA/light } \\
\hline BnaC04g15640D & & & 80.9 & -1.4 & -1.4 & & \\
\hline BnaA06g07470D & \multirow[t]{3}{*}{ AT1G13260 } & \multirow[t]{3}{*}{ RAV1 } & 85.5 & -2.4 & -2.5 & \multirow{3}{*}{$\begin{array}{l}\text { Ethylene-responsive transcription } \\
\text { factor RAV1 }\end{array}$} & \multirow[t]{3}{*}{ cold $/ \mathrm{NaCl}$} \\
\hline \multirow{2}{*}{$\begin{array}{l}\text { BnaAnng40580D } \\
\text { BnaCnng37790D }\end{array}$} & & & 84.6 & -3.0 & -2.4 & & \\
\hline & & & 84.9 & -1.4 & -1.7 & & \\
\hline BnaA06g24950D & AT5G67580 & TRB2 & 79.4 & -1.1 & -1.3 & Telomere repeat-binding factor 2 & cold/NaCl/drought \\
\hline
\end{tabular}

*Homology search was performed by comparing the predicted proteomes of $B$. napus and A. thaliana

\#Log2foldchange indicates the expression variation between the cold stressed plants and the control. Genes were collected only when they were differentially expressed in both 158A and SGDH284 in the same manner

${ }^{*}$ Stress elements were assigned based on the description of $A$. thaliana genes in STIFDB

we analyzed the gene expression change in cold stressed semi-winter type and winter type B. napus lines. Similar to the findings in other plants that about $10-15 \%$ genes were differentially expressed in response to cold stress [23-25], global modulation of gene expression was also observed in B. napus lines 158A and SGDH284 after low temperature exposure. Unexpectedly, despite of the high similarity in genetics between 158A and SGDH284, only $60 \%$ of the DEGs were shared. The shared DEGs indicated some common biochemical and physiological features of cold stressed plants, such as enhanced ability of stresses tolerance and inhibited photosynthesis. Cold acclimation accompanied inhibition of photosynthesis was observed in many plants, due to the effects of cold on electronic transport and carbon fixation, as well as the availability of free phosphate in the chloroplast, which could be assessed by the content of chlorophyll a [26]. In addition, further inspection of the about $40 \%$ line-specific DEGs demonstrated significantly varied gene regulatory mechanisms in cold stressed 158A and SGDH284. Stresses responsive related genes were enriched in both sets of line-specific DEGs, but were oppositely regulated in 158A and SGDH284. Similarly, biological processes related to the basic cellular activities were significantly inhibited in 158A but enhanced in SGDH284. This was consistent with the fact that growth of semi-winter type B. napus plants were more significantly inhibited than the winter cultivars once encountered extremely cold temperature in the field. Interestingly, histone $\mathrm{H} 2 \mathrm{~B}$ ubiquitination related genes were enriched in SGDH284 specific genes. Previous reports revealed that $\mathrm{H} 2 \mathrm{~B}$ monoubiquitination was related to the activation of transcription, which was related to the regulation of cuticle composition that could protect the plants from abiotic/biotic stresses, and also contributed to the plant growth fitness by modulating the expression of circadian clock genes [27-29]. It seems histone $\mathrm{H} 2 \mathrm{~B}$ ubiquitination played crucial roles in SGDH284 adaptation to low temperature survival based on this work, however, further investigations are needed for exploration of the exact roles and mechanisms.

Expression of previously characterized COR genes were checked in this work. Most of them were also upregulated in both semi-winter and winter type B. napus, which was consistent with the previous reports. But some of them, like $B n C O R 25$ and $h s p 90$, were not altered in expression in any line. We have noticed that, in contrast to a short-day photoperiod in this work, accumulation of $B n C O R 25$ and $h s p 90$ in cold stressed $B$. napus was detected under the $16 \mathrm{~h}$ light $/ 8 \mathrm{~h}$ dark cycle $[7,9]$. Thus further investigation is required to explore whether these differences is caused by the photoperiod.

Derived from the comparative genomics analysis in this work, members of particular TF families tended to be upor down-regulated in cold acclimation of B. napus, such as WRKY and GRF genes, respectively. WRKY genes were involved in plant response to kinds of environmental 
Table 3 Regulation based identification of key transcription factors involved in cold response of Brassica napus

\begin{tabular}{ll}
\hline Transcription factor $^{*}$ & Description $^{\#}$ \\
\hline BnaC07g43590D & two-component response regulator ARR10 $^{\text {BnaC07g07840D }}$ \\
& \\
transcription factor bHLH77 \\
BnaC01g10420D & transcription factor MYC4 \\
BnaAnng03730D & ethylene-responsive transcription factor ERF011
\end{tabular}

BnaA05g04450D

BnaA03g19970D

BnaA09g37540D

BnaA03g40080D

BnaA06g27900D

BnaCnng16520D

BnaC05g14070D

BnaA06g12480D

BnaA06g24950D

BnaC09g47560D

BnaA06g26010D

BnaC06g22430D

BnaC08g05600D

BnaC05g17700D

BnaA01g37250D

BnaA10g22560D

BnaA07g24230D ethylene-responsive transcription factor ERF034

transcription factor PIF4-like

transcription factor PIF5-like

transcription factor PIF7

ethylene-responsive transcription factor TINY

transcription factor DIVARICATA

REVEILLE 7

REVEILLE 7

telomere repeat-binding factor 2

transcription factor TCP21

transcription factor TCP7-like

bZIP transcription factor 44

bZIP transcription factor 60

transcription factor TGA3

protein indeterminate-domain 11

calmodulin-binding transcription activator 1

cyclic dof factor 5
Predicted function

NULL

GO:0015979 photosynthesis

GO:0019684 photosynthesis, light reaction

GO:0009186 deoxyribonucleoside diphosphate metabolic process

NULL

GO:1901606 alpha-amino acid catabolic process GO:0006544 glycine metabolic process

GO:1901565 organonitrogen compound catabolic process

NULL

GO:0015979 photosynthesis GO:0009853 photorespiration GO:0015977 carbon fixation

GO:0009853 photorespiration GO:0015977 carbon fixation GO:0015979 photosynthesis

GO:0015979 photosynthesis

GO:0006091 generation of precursor metabolites and energy

GO:0019684 photosynthesis, light reaction

NULL

NULL

GO:0000160 phosphorelay signal transduction system

GO:0050896 response to stimulus

GO:0007623 circadian rhythm

GO:0043086 negative regulation of catalytic activity

GO:0010035 response to inorganic substance GO:0009892 negative regulation of metabolic process

GO:0009845 seed germination

GO:0071103 DNA conformation change

G0:0006091 generation of precursor metabolites and energy

GO:0033014 tetrapyrrole biosynthetic process GO:0046148 pigment biosynthetic process GO:0033013 tetrapyrrole metabolic process

GO:0015995 chlorophyll biosynthetic process GO:0018130 heterocycle biosynthetic process GO:0019438 aromatic compound biosynthetic process

GO:0015979 photosynthesis

GO:0018298 protein-chromophore linkage GO:0009773 photosynthetic electron transport in photosystem I

NULL

NULL

GO:0016114 terpenoid biosynthetic process GO:0006720 isoprenoid metabolic process GO:0008610 lipid biosynthetic process

GO:0080022 primary root development GO:0016052 carbohydrate catabolic process GO:0009664 plant-type cell wall organization

GO:0006355 regulation of transcription, DNA-templated GO:0008643 carbohydrate transport 
Table 3 Regulation based identification of key transcription factors involved in cold response of Brassica napus (Continued)

\begin{tabular}{lll}
\hline Transcription factor $^{*}$ & Description $^{\#}$ & Predicted function \\
\hline BnaAnng34260D & CBF-7 & GO:0016998 cell wall macromolecule catabolic process \\
& & GO:0046348 amino sugar catabolic process \\
BnaC08g04820D & GO:0006040 amino sugar metabolic process \\
BnaC07g29370D & NULL \\
& $\begin{array}{l}\text { ethylene-responsive transcription factor RAP2-1 } \\
\text { factor SHINE 3 }\end{array}$ & GO:0015979 photosynthesis \\
& & GO:0018298 protein-chromophore linkage \\
BnaC07g13550D & NAC domain-containing protein 13 & incompatible interaction
\end{tabular}

*Transcription factors were collected if their potential targets were over-represented in the total differentially expressed genes in both $158 \mathrm{~A}$ and SGDH284 \#Possible function of these genes in cold stressed B. napus were predicted by analysis of their differentially expressed targets (top three GO terms were shown). Bold identifiers indicated the down-regulated genes, while the others were up-regulated genes in cold stressed plants

stresses [30, 31], and up-regulation of some WRKY genes in $B$. napus response to multiple stresses was previously observed [32,33]. In the semi-winter type line 158A, all the differentially expressed WRKY genes were upregulated, indicating their crucial roles in cold tolerance of 158A. Moreover, GRFs usually played a role in the regulation of cell expansion in leaf and cotyledon [34], and in a semi-winter type B. napus zy036, the role of GRF2 in enhancing seed oil production was observed, which possibly worked by regulating cell number and plant photosynthesis [35]. Herein, down-regulation of GRF2a and GRF2b (BnaA01g00300D/BnaC07g46760D) was observed in both semi-winter and winter type lines, indicating their conserved roles in regulation of photosynthesis. More downregulated GRF genes were found in 158A, which coincided with the phenotype that winter type B. napus usually exhibit stronger physiological activity after cold stress and that the growth of semi-winter type $B$. napus lines were more severely restricted by cold condition. However, whether these facts are directly associated with the different cold responsive mechanisms between semi-winter and winter type rapeseeds remains to be cleared.

56 and 25 cold responsive TFs were subsequently predicted based on homology search and enrichment analysis, respectively, which were regulated in the same manner in both semi-winter and winter type B. napus lines. Inferred from the functional analysis of target genes and the reconstructed transcriptional cascades, several TFs were assumed to be of great importance in modulating the gene expression to enhance the cold tolerance of B. napus, e.g., CBF7, BHLH77, TCP7, TCP21 and PIF7, including some well characterized COR genes like CBF7 and PIF7, indicating our strategy was an effective method for exploration of key regulators.

\section{Conclusions}

Extensive biochemical and physiological adjustment and gene expression modulation are required for plants in

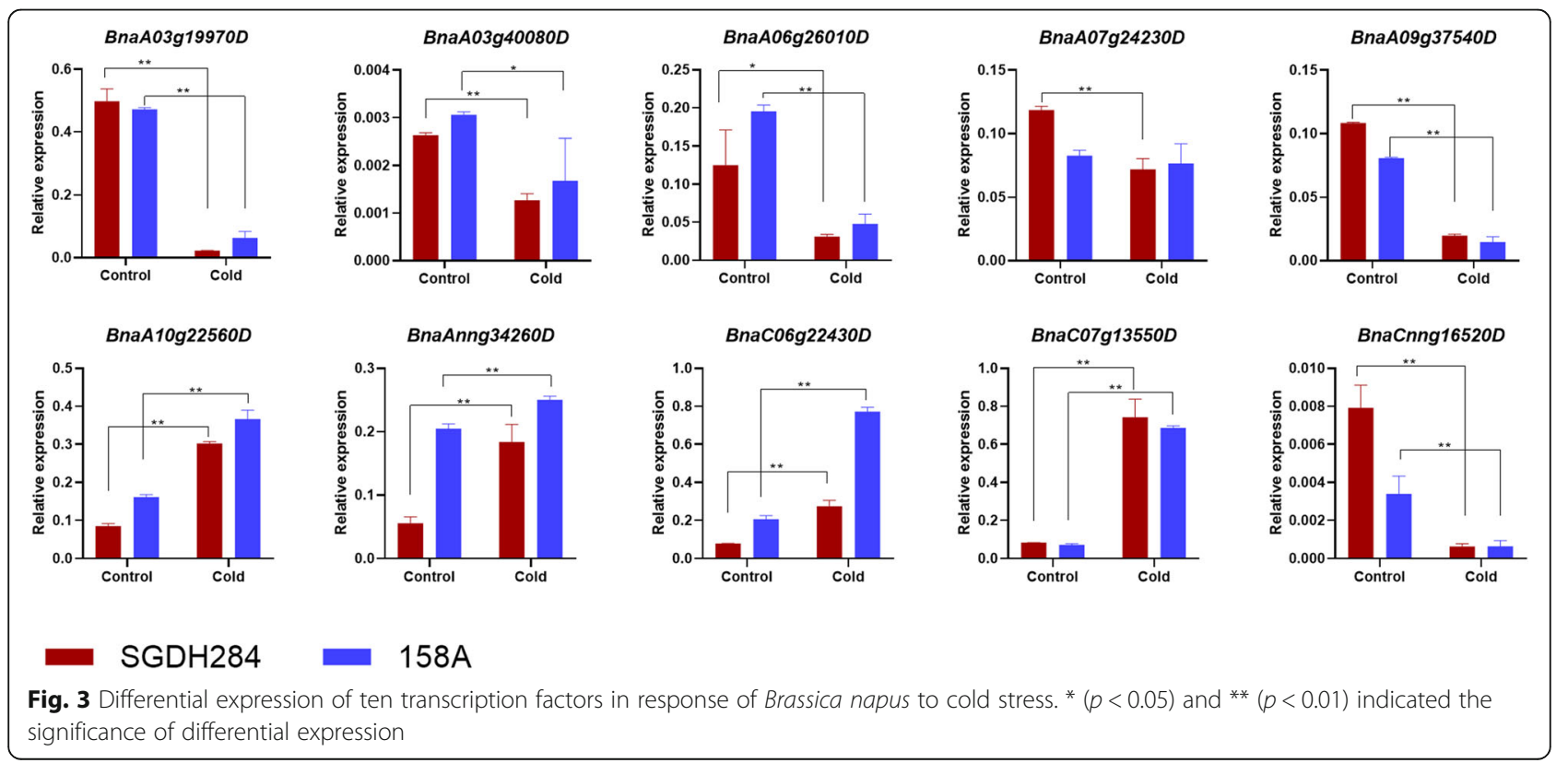




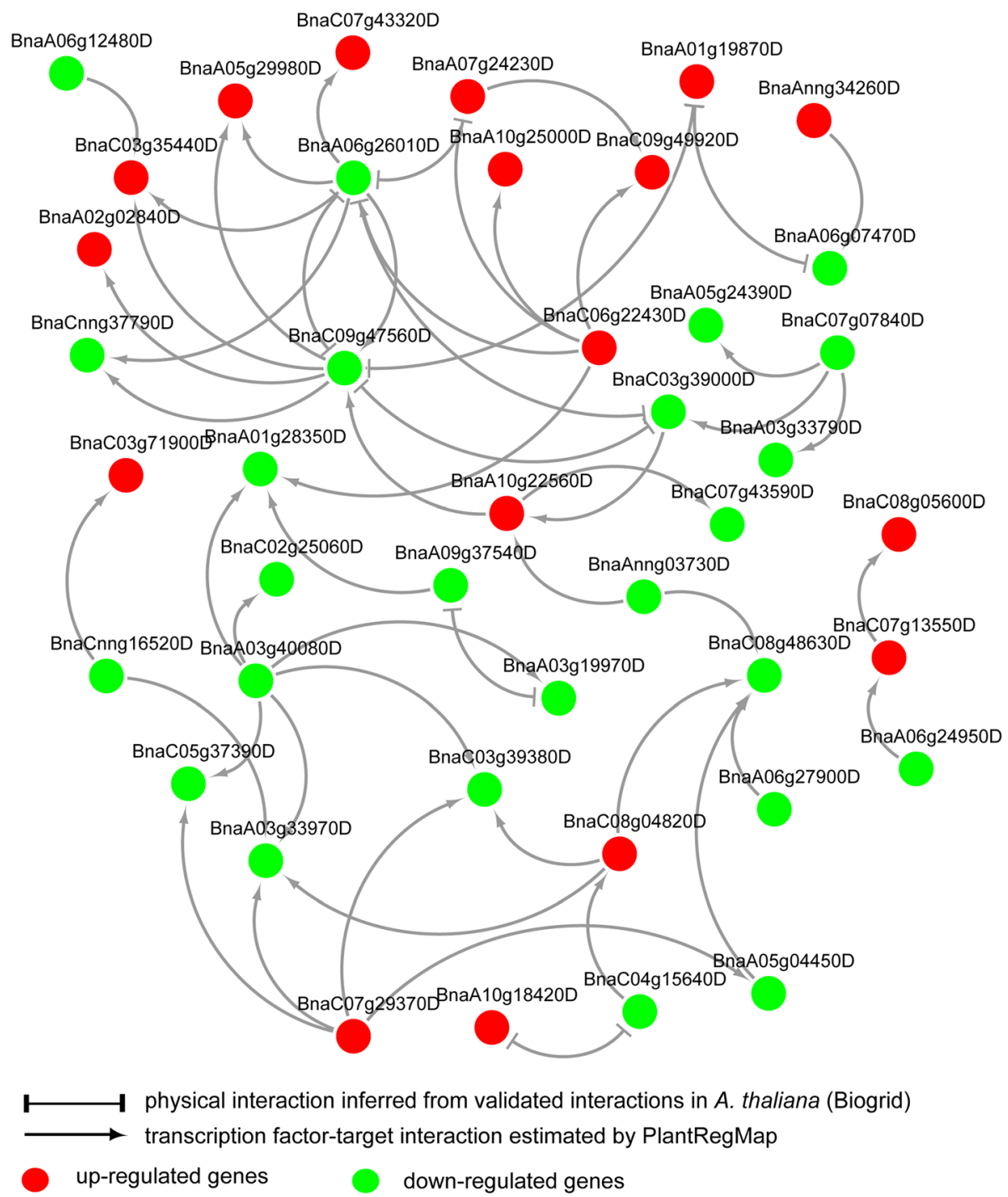

Fig. 4 Interaction networks of identified cold responsive transcription factors in Brassica napus. Transcription factors were identified by using homology search and TF enrichment analysis. Interactions were estimated based on the protein-protein interactions in Arabidopsis thaliana and the reciprocal best hit pairs between $A$. thaliana and B. napus, as well as the regulations predicted in B. napus. All these genes were regulated in the same manner in 158A and SGDH284

response to low temperature. Some genes and pathways play similar roles across plant species, such as CBFs and photosynthesis/chloroplast related genes [36]. However, widespread variation in gene expression in cold stressed plants was also observed, which was largely ascribed to the inherent genetic difference. In order to identify the conserved genes and pathways involved in B. napus response to cold stress, we compared the transcriptional changes in semi-winter type line 158A and winter type line SGDH284. Enhanced stresses response mechanisms and inhibited photosynthesis were observed in both lines, indicating the common features of $B$. napus cold acclimation were conserved within species. Nevertheless, we also found that about 40\% DEGs were line specific, suggesting the remodeling of regulatory mechanisms occurred during environmental adaptation. Moreover, involvement about eighty key TFs in B. napus cold response were predicted by an integrated strategy. These regulators covered main characterized cold response mechanisms and were responsible for expression variation of a great number of genes, thus provided a valuable collection for comprehensive understanding of the stress responsive mechanisms in B. napus.

Apart from transcriptomics analysis performed in this work and elsewhere, the other strategies like genomewide association study (GWAS) have also played crucial roles in identification of genes related to agricultural traits [37-39], as well as the locus/genes involved in 
plants response to varied environmental stresses like low temperature [40-42]. Considering the characteristics of transcriptomes based analysis, e.g., they are usually used to assess tissue-specific and stage-specific regulations, application of multi-dimensional research methods including transcriptomics, GWAS and the integrated strategy such as expression quantitative trait loci (eQTL) are therefore expected for systematically depiction of the cold stress mechanisms in B. napus in future.

\section{Methods}

\section{Plant materials and growth conditions}

Brassica napus SGDH284 and 158A, two DH lines selected in our lab from microspore culture of winter rapeseed Sollux and semi-winter rapeseed zhongyou 9988, respectively, were used in this study. Sollux was kindly provided by Dr. Xiyuan Ni from Zhejiang Academy of Agricultural Sciences, and zhongyou 9988 is a commercial variety in China selected by Oil Institute of Chinese Academy of Agricultural Sciences. Seeds were planted in $4 \times 8$ hole flowerpots $(54 \times 28 \times 7 \mathrm{~cm})$ containing soil substrates and pearlite with a ratio of 3:1. The flowerpots were transferred into a plant growth chamber (Percival E-36 L) under a short-day photoperiod (10-h light/ 14-h dark) and a humidity of $60 \%$ at $25^{\circ} \mathrm{C}$ and were grown for 4 weeks since germination. For cold treatment two trays of the seedlings of each lines were transferred to another plant growth chamber at $4{ }^{\circ} \mathrm{C}$ under the same photoperiod and humidity for 7 days. Another four trays of seedlings were set as the control group and were left at the same plant growth chamber with the same light and temperature condition. After treatments, the fully expanded first leaves of 6-8 plants from the same trays were collected and pooled as one sample. The leaves were frozen in liquid nitrogen as soon as they were cut down from the plants. Then the samples were stored at $-80^{\circ} \mathrm{C}$ for further analysis.

\section{RNA extraction and RNA-seq}

Total RNA was isolated using the TRIzol regent (Invitrogen, Carlsbad, USA) according to the manufacturer's instructions. The RNA quantity and quality were checked by gel electrophoresis and a DU800 spectrophotometer (BECKMAN, USA). A total of $50 \mu \mathrm{g}$ RNA $(1000 \mathrm{ng} / \mu \mathrm{L})$ for each sample was used for RNA sequencing. The Illumina NextSeq 500 platform was applied for RNA-seq. Eight libraries were constructed and paired-end sequencing was performed according to the manufacturer's instructions (TruSeq RNA Sample Prep Kit, Illumina). Shortly, magnetic beads with oligo poly $(\mathrm{T})$ attached were used for purifying the mRNA, which were cut into short fragments of about $375 \mathrm{bp}$ in fragmentation buffer. Then the purified fragmented mRNA were converted into double-stranded cDNA and adapters were added to both end of the short fragments. AMPure XP beads were used to remove the unsuitable fragments and by PCR amplification the sequencing libraries were constructed. The libraries were normalized and loaded to the Illumina NextSeq 500 platform (Shanghai Personal Biotechnology Co Ltd, Shanghai, China) for sequencing after being checked with Pico green staining (PicoGreen dsDNA assay kit, Invitrogen, P7589) and fluorospectrophotometry (Quantiflour-ST fluorometer, Promega, E6090), and quantified with Agilent 2100 (Agilent 2100 Bioanalyzer, Agilent 2100; Agilent High Sensitivity DNA Kit, Agilent, 5067-4626).

\section{Identification of differentially expressed genes}

Quality control of raw reads generated from Illumina platform were performed by using Trimmomatic (version 0.33) (LEADING:20, TRAILING:20 SLIDINGWINDOW:4:20, MINLEN:25) [43]. Clean reads were then mapped to the reference genome of Brassica napus from EnsemblPlants (http://plants.ensembl.org, release 42) with TopHat (v2.1.0) (default setting) [44]. Cufflinks (v2.2.1) was used to reconstruct the transcriptomes and calculated the expression level. Only genes with their FPKM (Fragments Per Kilo bases per Million reads) $\geq 1$ in at least one samples were collected for further analysis. HTseq-count (version 0.11.2) was used to calculated the number of short reads mapped to the characterized gene locus and DEseq2 was used for identification of differentially expressed genes (DEGs) (foldchange $\geq 2$ and $p$-value $\leq 0.05)[45,46]$.

\section{Functional enrichment analysis}

GO enrichment analysis in this work was carried out using the tools provided by PlantRegMap (http://plantregmap.cbi.pku.edu.cn) [47]. The online server REViGO (http://revigo.irb.hr/) was used to remove the redundancy of GO terms [48].

\section{Identification of cold responsive transcription factors}

Homology based identification of cold responsive TFs was performed by comparing the $B$. napus genes to the stresses responsive TFs in $A$. thaliana with the BLASTP tool (cutoff identity 50\% and coverage 50\% for both query and hit sequences in length). Stresses responsive TFs in A. thaliana were retrieved and downloaded from the STIFDB (http://caps.ncbs.res.in/stifdb2/) [17]. In addition, TF enrichment analysis was performed through PlantRegMap with the tool 'TF Enrichment' (cutoff pvalue 0.05) [47]. Briefly, transcriptional regulations in $B$. napus were firstly identified based on the genome-wide analysis of functional transcription factor binding sites (FunTFBS). Subsequently, Fisher's exactly test was performed to identify the TFs with their targets overrepresented in the total DEGs in the cold stressed 158A and SGDH284, respectively. 


\section{Interaction networks of cold responsive TFs}

Firstly, interactions between A. thaliana proteins were retrieved from the BioGRID [49]. Protein-protein interactions for TFs in B. napus were then predicted according to the estimated BLAST reciprocal best hits between A. thaliana and B. napus in PlantRegMap. Potential regulations between the TFs in B. napus were retrieved from PlantRegMap (FunTFBS method), which were then merged with the predicted protein-protein interactions using in house developed perl scripts. Cytoscape (3.4.0) was used to display and analyze the constructed networks [50].

\section{Quantitative real-time PCR analysis}

To validate the expression profiles of genes, ten randomly selected DEGs were used to perform Quantitative Real-Time PCR (qRT-PCR) analysis. Three samples of each treatment were harvested for total RNAs extraction, which were different from those used in RNA-seq. After reverse-transcribed with FastQuant RT Kit (TIANGEN Biotech, Beijing, China), the qRT-PCR reactions were performed with THUNDERBIRD SYBR qPCR Mix (TOYOBO, Osaka, Japan) and a Mastercycler ep realplex (Eppendorf, Germany) according to the manufacturer's instructions. The thermal cycling conditions were as follows: $94^{\circ} \mathrm{C}$ for $2 \mathrm{~min}$, followed by 40 cycles of $10 \mathrm{~s}$ at $94^{\circ} \mathrm{C}$ and $20 \mathrm{~s}$ at $60^{\circ} \mathrm{C}$, then $72{ }^{\circ} \mathrm{C}$ for $20 \mathrm{~s}$. All reactions were performed in triplicate, and the actin 7 was used as an internal reference gene (accession number: Bra028615) [51]. The relative gene expression levels were calculated by using the $2-\Delta \Delta \mathrm{Ct}$ method [52].

\section{Statistics}

Elsewise specified, chi-square test was used in this work to calculate the significance of gene number variation between different groups.

\section{Supplementary information}

Supplementary information accompanies this paper at https://doi.org/10. 1186/s12870-020-2253-5.

Additional file 1: Table S1. Statistics of RNA-seq and short reads mapping. Table S2. Significantly varied GO terms between 158A and SGDH284 for line specific differentially expressed genes. Table S3. Statistics of differentially expressed transcription factors in 158A and SGDH284. Table S4. Enrichment analysis of cold responsive transcription factors in 158A and SGDH284. Table S5. Primer pairs used to detect the expression of selected transcription factors.

\section{Abbreviations}

CBFs: Cold-binding transcription factors; COR: Cold-responsive; DEGs: Differentially expressed genes; DREBs: Dehydration responsive element binding factors; GRFs: Growth-regulation factors.; TFs: Transcription factors

\section{Acknowledgments}

The authors thank Dr. Xiyuan Ni from Zhejiang Academy of Agricultural Sciences for providing seeds of Sollux.

\section{Author contributions}

Conceptualization, L.K., Y.S. and Z.F.; methodology, W.L. and Z.F.; software, D.Y.; validation, L.K., W.L., Z.F. and D.Y.; formal analysis, J.W. and D.Y.; investigation, L.K. W.Y., J.G. and J.C.; resources, W.L., Y.S. and Z.F.; data curation, D.Y.;

writing —original draft preparation, L.K., Z.F. and D.Y.; writing—review and editing, D.Y. and Y.S.; visualization, L.K.; supervision, Y.S.; project administration, L.K., Y.S. and Z.F.; funding acquisition, L.K., W.L., D.Y. and Z.F. All authors read and approved the final manuscript.

\section{Funding}

This research was funded by Teamwork Construction in Technology Innovations of AAAS, grant number 15C0207, Breeding for New GM Oilseed Hybrids downstream Yangtze River, grant number 2018ZX08020001-005, Hangzhou Science and Technology Development Plan of Zhejiang Province, grant number 20160432B06 and Science Foundation of Zhejiang Sci-Tech University, grant number 19042398-Y. The funding agencies had no role in research design, data collection and analysis, or manuscript writing.

\section{Availability of data and materials}

Raw data generated by this work is available in BIGD under the project PRJCA001621 (https://bigd.big.ac.cn/bioproject/browse/PRJCA001621).

Ethics approval and consent to participate

Not applicable.

\section{Consent for publication}

Not applicable.

\section{Competing interests}

The authors declare that they have no competing interests.

\section{Author details}

'Plant Genomics \& Molecular Improvement of Colored Fiber Lab, College of Life Sciences and Medicine, Zhejiang Sci-Tech University, Hangzhou 310018, China. ${ }^{2}$ Crop Institute, Anhui Academy of Agricultural Sciences, Hefei 230031,

China. ${ }^{3}$ Wenzhou - Kean University, Wenzhou 325060, China.

Received: 5 November 2019 Accepted: 16 January 2020

Published online: 06 February 2020

\section{References}

1. Thomashow MF. PLANT COLD ACCLIMATION: freezing tolerance genes and regulatory mechanisms. Annu Rev Plant Physiol Plant Mol Biol. 1999:50:571-99.

2. Shi Y, Ding Y, Yang S. Molecular regulation of CBF signaling in cold acclimation. Trends Plant Sci. 2018;23(7):623-37.

3. Liu Y, Dang P, Liu L, He C. Cold acclimation by the CBF-COR pathway in a changing climate: lessons from Arabidopsis thaliana. Plant Cell Rep. 2019; 38(5):511-9.

4. Shi $Y$, Ding $Y$, Yang S. Cold signal transduction and its interplay with phytohormones during cold acclimation. Plant Cell Physiol. 2015;56(1):7-15.

5. Stefanowska M, Kuras M, Kacperska A. Low temperature-induced modifications in cell ultrastructure and localization of phenolics in winter oilseed rape (Brassica napus L. var. oleifera L.) leaves. Ann Bot. 2002;90(5): 637-45.

6. Weretilnyk E, Orr W, White TC, lu B, Singh J. Characterization of three related low-temperature-regulated cDNAs from winter Brassica napus. Plant Physiol. 1993;101(1):171-7.

7. Chen L, Zhong H, Ren F, Guo QQ, Hu XP, Li XB. A novel cold-regulated gene, COR25, of Brassica napus is involved in plant response and tolerance to cold stress. Plant Cell Rep. 2011;30(4):463-71.

8. Orr W, White TC, lu B, Robert L, Singh J. Characterization of a lowtemperature-induced cDNA from winter Brassica napus encoding the 70 kDa subunit of tonoplast ATPase. Plant Mol Biol. 1995;28(5):943-8.

9. Krishna P, Sacco M, Cherutti JF, Hill S. Cold-induced accumulation of hsp90 transcripts in Brassica napus. Plant Physiol. 1995;107(3):915-23.

10. Gao MJ, Allard G, Byass L, Flanagan AM, Singh J. Regulation and characterization of four CBF transcription factors from Brassica napus. Plant Mol Biol. 2002;49(5):459-71.

11. Savitch LV, Allard G, Seki M, Robert LS, Tinker NA, Huner NP, Shinozaki K, Singh J. The effect of overexpression of two Brassica CBF/DREB1-like 
transcription factors on photosynthetic capacity and freezing tolerance in Brassica napus. Plant Cell Physiol. 2005:46(9):1525-39.

12. Du C, Hu K, Xian S, Liu C, Fan J, Tu J, Fu T. Dynamic transcriptome analysis reveals AP2/ERF transcription factors responsible for cold stress in rapeseed (Brassica napus L.). Mol Genet Genomics. 2016;291(3):1053-67.

13. Wang B, Guo X, Wang C, Ma J, Niu F, Zhang H, Yang B, Liang W, Han F, Jiang YQ. Identification and characterization of plant-specific NAC gene family in canola (Brassica napus L.) reveal novel members involved in cell death. Plant Mol Biol. 2015;87(4-5):395-411.

14. Megha S, Basu U, Joshi RK, Kav NNV. Physiological studies and genomewide microRNA profiling of cold-stressed Brassica napus. Plant Physiol Biochem. 2018;132:1-17.

15. Shea DJ, Nishida N, Takada S, Itabashi E, Takahashi S, Akter A, Miyaji N, Osabe K, Mehraj H, Shimizu M, et al. Long noncoding RNAs in Brassica rapa L. following vernalization. Scientific reports. 2019;9(1):9302.

16. Orr W, Iu B, White TC, Robert LS, Singh J. Complementary DNA sequence of a low temperature-induced Brassica napus gene with homology to the Arabidopsis thaliana kin1 gene. Plant Physiol. 1992;98(4):1532-4.

17. Naika M, Shameer K, Mathew OK, Gowda R, Sowdhamini R. STIFDB2: an updated version of plant stress-responsive transcription factor database with additional stress signals, stress-responsive transcription factor binding sites and stress-responsive genes in Arabidopsis and rice. Plant Cell Physiol. 2013;54(2):e8.

18. Hegedus D, Yu M, Baldwin D, Gruber M, Sharpe A, Parkin I, Whitwill S, Lydiate D. Molecular characterization of Brassica napus NAC domain transcriptional activators induced in response to biotic and abiotic stress. Plant Mol Biol. 2003;53(3):383-97.

19. Kuno N, Moller SG, Shinomura T, Xu X, Chua NH, Furuya M. The novel MYB protein EARLY-PHYTOCHROME-RESPONSIVE1 is a component of a slave circadian oscillator in Arabidopsis. Plant Cell. 2003;15(10):2476-88.

20. Pruneda-Paz JL, Breton G, Para A, Kay SA. A functional genomics approach reveals $\mathrm{CHE}$ as a component of the Arabidopsis circadian clock. Science. 2009;323(5920):1481-5.

21. Iglesias-Fernandez R, Barrero-Sicilia C, Carrillo-Barral N, Onate-Sanchez L, Carbonero P. Arabidopsis thaliana bZIP44: a transcription factor affecting seed germination and expression of the mannanase-encoding gene AtMAN7. Plant J. 2013;74(5):767-80.

22. Fornara F, Panigrahi KC, Gissot L, Sauerbrunn N, Ruhl M, Jarillo JA, Coupland G. Arabidopsis DOF transcription factors act redundantly to reduce CONSTANS expression and are essential for a photoperiodic flowering response. Dev Cell. 2009;17(1):75-86.

23. Lee $\mathrm{BH}$, Henderson DA, Zhu JK. The Arabidopsis cold-responsive transcriptome and its regulation by ICE1. Plant Cell. 2005;17(11):3155-75.

24. Zhang $T$, Zhao $X$, Wang W, Pan Y, Huang L, Liu X, Zong Y, Zhu L, Yang D, Fu B. Comparative transcriptome profiling of chilling stress responsiveness in two contrasting rice genotypes. PLoS One. 2012;7(8):e43274.

25. Waters AJ, Makarevitch I, Noshay J, Burghardt LT, Hirsch CN, Hirsch CD, Springer NM. Natural variation for gene expression responses to abiotic stress in maize. Plant J. 2017:89(4):706-17.

26. Nievola CC, Carvalho CP, Carvalho V, Rodrigues E. Rapid responses of plants to temperature changes. Temperature. 2017:4(4):371-405.

27. Feng J, Shen WH. Dynamic regulation and function of histone monoubiquitination in plants. Front Plant Sci. 2014;5:83.

28. Himanen K, Woloszynska M, Boccardi TM, De Groeve S, Nelissen H, Bruno L, Vuylsteke M, Van Lijsebettens M. Histone H2B monoubiquitination is required to reach maximal transcript levels of circadian clock genes in Arabidopsis. Plant Journal. 2012;72(2):249-60.

29. Menard R, Verdier G, Ors M, Erhardt M, Beisson F, Shen WH. Histone H2B monoubiquitination is involved in the regulation of cutin and wax composition in Arabidopsis thaliana. Plant cell Physiol. 2014;55(2):455-66.

30. Banerjee A, Roychoudhury A. WRKY proteins: signaling and regulation of expression during abiotic stress responses. Scientific World J. 2015;2015: 807560

31. Eulgem T, Rushton PJ, Robatzek S, Somssich IE. The WRKY superfamily of plant transcription factors. Trends Plant Sci. 2000;5(5):199-206.

32. He Y, Mao S, Gao Y, Zhu L, Wu D, Cui Y, Li J, Qian W. Genome-wide identification and expression analysis of WRKY transcription factors under multiple stresses in Brassica napus. PLoS One. 2016;11(6):e0157558.

33. Yang B, Jiang $Y$, Rahman MH, Deyholos MK, Kav NN. Identification and expression analysis of WRKY transcription factor genes in canola (Brassica napus L.) in response to fungal pathogens and hormone treatments. BMC Plant Biol. 2009:9:68.

34. Kim JH, Choi D, Kende $H$. The AtGRF family of putative transcription factors is involved in leaf and cotyledon growth in Arabidopsis. Plant J : for cell and molecular biology. 2003;36(1):94-104.

35. Liu J, Hua W, Yang HL, Zhan GM, Li RJ, Deng LB, Wang XF, Liu GH, Wang HZ. The BnGRF2 gene (GRF2-like gene from Brassica napus) enhances seed oil production through regulating cell number and plant photosynthesis. J Exp Bot. 2012;63(10):3727-40

36. Kenchanmane Raju SK, Barnes AC, Schnable JC, Roston RL. Low-temperature tolerance in land plants: are transcript and membrane responses conserved? Plant Sci : an international journal of experimental plant biology. 2018;276: 73-86.

37. Li F, Chen B, Xu K, Wu J, Song W, Bancroft I, Harper AL, Trick M, Liu S, Gao $G$, et al. Genome-wide association study dissects the genetic architecture of seed weight and seed quality in rapeseed (Brassica napus L.). DNA Res : an international journal for rapid publication of reports on genes and genomes. 2014;21(4):355-67.

38. Xu L, Hu K, Zhang Z, Guan C, Chen S, Hua W, Li J, Wen J, Yi B, Shen J, et al. Genome-wide association study reveals the genetic architecture of flowering time in rapeseed (Brassica napus L.). DNA Res. 2016;23(1):43-52.

39. Arab MM, Marrano A, Abdollahi-Arpanahi R, Leslie CA, Askari H, Neale DB, Vahdati K. Genome-wide patterns of population structure and association mapping of nut-related traits in Persian walnut populations from Iran using the Axiom J. regia 700K SNP array. Sci Rep. 2019;9(1):6376.

40. Hu G, Li Z, Lu Y, Li C, Gong S, Yan S, Li G, Wang M, Ren H, Guan H, et al. Genome-wide association study identified multiple genetic loci on chilling resistance during germination in maize. Sci Rep. 2017;7(1):10840.

41. Liu C, Chen K, Zhao X, Wang X, Shen C, Zhu Y, Dai M, Qiu X, Yang R, Xing $D$, et al. Identification of genes for salt tolerance and yield-related traits in rice plants grown hydroponically and under saline field conditions by genome-wide association study. Rice. 2019;12(1):88.

42. Wrucke DF, Mamidi S, Rahman M. Genome-wide association study for frost tolerance in canola (Brassica napus L.) under field conditions. J Plant Biochem Biotechnol. 2019;28(2):211-22

43. Bolger AM, Lohse M, Usadel B. Trimmomatic: a flexible trimmer for Illumina sequence data. Bioinformatics. 2014;30(15):2114-20.

44. Trapnell C, Roberts A, Goff L, Pertea G, Kim D, Kelley DR, Pimentel H, Salzberg SL, Rinn JL, Pachter L. Differential gene and transcript expression analysis of RNA-seq experiments with TopHat and cufflinks. Nat Protoc. 2012;7(3):562-78.

45. Anders S, Pyl PT, Huber W. HTSeq--a Python framework to work with highthroughput sequencing data. Bioinformatics. 2015;31(2):166-9.

46. Love Ml, Huber W, Anders S. Moderated estimation of fold change and dispersion for RNA-seq data with DESeq2. Genome Biol. 2014;15(12):550.

47. Jin J, Tian F, Yang DC, Meng YQ, Kong L, Luo J, Gao G. PlantTFDB 4.0: toward a central hub for transcription factors and regulatory interactions in plants. Nucleic Acids Res. 2017:45(D1):D1040-5.

48. Supek F, Bosnjak M, Skunca N, Smuc T. REVIGO summarizes and visualizes long lists of gene ontology terms. PLoS One. 2011;6(7):e21800.

49. Oughtred R, Stark C, Breitkreutz BJ, Rust J, Boucher L, Chang C, Kolas N, O'Donnell L, Leung G, McAdam R, et al. The BioGRID interaction database: 2019 update. Nucleic Acids Res. 2019;47(D1):D529-41.

50. Shannon P, Markiel A, Ozier O, Baliga NS, Wang JT, Ramage D, Amin N, Schwikowski B, Ideker T. Cytoscape: a software environment for integrated models of biomolecular interaction networks. Genome Res. 2003;13(11): 2498-504.

51. Wang Z, Chen Y, Fang H, Shi H, Chen K, Zhang Z, Tan X. Selection of reference genes for quantitative reverse-transcription polymerase chain reaction normalization in Brassica napus under various stress conditions. Mol Genet Genomics. 2014;289(5):1023-35.

52. Livak KJ, Schmittgen TD. Analysis of relative gene expression data using real-time quantitative PCR and the $2(-$ Delta Delta $C(T))$ method. Methods. 2001;25(4):402-8.

\section{Publisher's Note}

Springer Nature remains neutral with regard to jurisdictional claims in published maps and institutional affiliations. 